\title{
GAN-Based Multiple Adjacent Brain MRI Slice Reconstruction for Unsupervised Alzheimer's Disease Diagnosis
}

\author{
Changhee $\operatorname{Han}^{1,2,3}(\varangle)$, Leonardo Rundo ${ }^{4,5}$, Kohei Murao ${ }^{1}$, \\ Zoltán Ádám Milacski ${ }^{6}$, Kazuki Umemoto ${ }^{7}$, Evis Sala ${ }^{4,5}$, \\ Hideki Nakayama ${ }^{3,8}$, and Shin'ichi Satoh ${ }^{1}$ \\ 1 Research Center for Medical Big Data, \\ National Institute of Informatics, Tokyo, Japan \\ han@nlab.ci.i.u-tokyo.ac.jp, \\ 2 LPixel Inc., Tokyo, Japan \\ ${ }^{3}$ Graduate School of Information Science and Technology, \\ The University of Tokyo, Tokyo, Japan \\ 4 Department of Radiology, University of Cambridge, Cambridge, United Kingdom \\ ${ }^{5}$ Cancer Research UK Cambridge Centre, Cambridge, United Kingdom \\ 6 Department of Artificial Intelligence, \\ ELTE Eötvös Loránd University, Budapest, Hungary \\ 7 Department of Rehabilitation Medicine, \\ Juntendo University School of Medicine, Tokyo, Japan \\ 8 International Research Center for Neurointelligence (WPI-IRCN), The University \\ of Tokyo Institutes for Advanced Study, The University of Tokyo, Tokyo, Japan
}

\begin{abstract}
Unsupervised learning can discover various unseen diseases, relying on large-scale unannotated medical images of healthy subjects. Towards this, unsupervised methods reconstruct a single medical image to detect outliers either in the learned feature space or from high reconstruction loss. However, without considering continuity between multiple adjacent slices, they cannot directly discriminate diseases composed of the accumulation of subtle anatomical anomalies, such as Alzheimer's Disease (AD). Moreover, no study has shown how unsupervised anomaly detection is associated with disease stages. Therefore, we propose a twostep method using Generative Adversarial Network-based multiple adjacent brain MRI slice reconstruction to detect $\mathrm{AD}$ at various stages: (Reconstruction) Wasserstein loss with Gradient Penalty $+\ell_{1}$ loss - trained on 3 healthy slices to reconstruct the next 3 ones - reconstructs unseen healthy/AD cases; (Diagnosis) Average/Maximum loss (e.g., $\ell_{2}$ loss) per scan discriminates them, comparing the reconstructed/ground truth images. The results show that we can reliably detect $\mathrm{AD}$ at a very early stage with Area Under the Curve (AUC) 0.780 while also detecting AD at a late stage much more accurately with AUC 0.917; since our method is fully unsupervised, it should also discover and alert any anomalies including rare disease.
\end{abstract}

Keywords: Generative adversarial networks · Alzheimer's disease diagnosis - Unsupervised anomaly detection - Brain MRI reconstruction. 


\section{Introduction}

Deep Learning can achieve accurate computer-assisted diagnosis when large-scale annotated training samples are available. In medical imaging, unfortunately, preparing such massive annotated datasets is often unfeasible; to tackle this important problem, researchers have proposed various data augmentation techniques, including Generative Adversarial Network (GAN)-based ones [1 2,3 4,5]. However, even exploiting these techniques, supervised learning still requires many images with pathological features, even for rare disease, to make a reliable diagnosis; nevertheless, it can only detect already-learned specific pathologies. In this regard, as physicians notice previously unseen anomaly examples using prior information on healthy body structure, unsupervised anomaly detection methods leveraging only large-scale healthy images can discover and alert unseen disease when their generalization fails.

Towards this, researchers reconstructed a single medical image via GANs [6], AutoEncoders (AEs) [7, or combining them, since GANs can generate realistic images and AEs, especially Variational AEs, can directly map data onto its latent representation [8]; then, unseen images were scored by comparing them with reconstructed ones to discriminate a pathological image distribution (i.e., outliers either in the learned feature space or from high reconstruction loss). However, those single image reconstruction methods mainly target diseases easy-to-detect from a single image even for non-expert human observers, such as glioblastoma on Magnetic Resonance (MR) images [8] and lung cancer on Computed Tomography images [7]. Without considering continuity between multiple adjacent images, they cannot directly discriminate diseases composed of the accumulation of subtle anatomical anomalies, such as Alzheimer's Disease (AD). Moreover, no study has shown so far how unsupervised anomaly detection is associated with disease stages. We thus propose a two-step method using GAN-based multiple adjacent brain MRI slice reconstruction to detect $\mathrm{AD}$ at various stages (Fig. 1): (Reconstruction) Wasserstein loss with Gradient Penalty (WGAN-GP) [910]+ $\ell_{1}$ loss - trained on 3 healthy brain axial MRI slices to reconstruct the next 3 ones - reconstructs unseen healthy/AD cases; (Diagnosis) Average/Maximum loss (e.g., $\ell_{2}$ loss) per scan discriminates them, comparing the reconstructed and ground truth images.

Contributions. Our main contributions are as follows:

- MRI Slice Reconstruction: This first multiple MRI slice reconstruction approach can predict the next 3 brain MRI slices from the previous 3 ones only for unseen images similar to training data by combining WGAN-GP and $\ell_{1}$ loss.

- Unsupervised Anomaly Detection: This first unsupervised anomaly detection across different disease stages reveals that, like physicians' way of diagnosis, massive healthy data can reliably aid early diagnosis, such as of MCI, while also detecting late-stage disease much more accurately by discriminating with $\ell_{2}$ loss. 

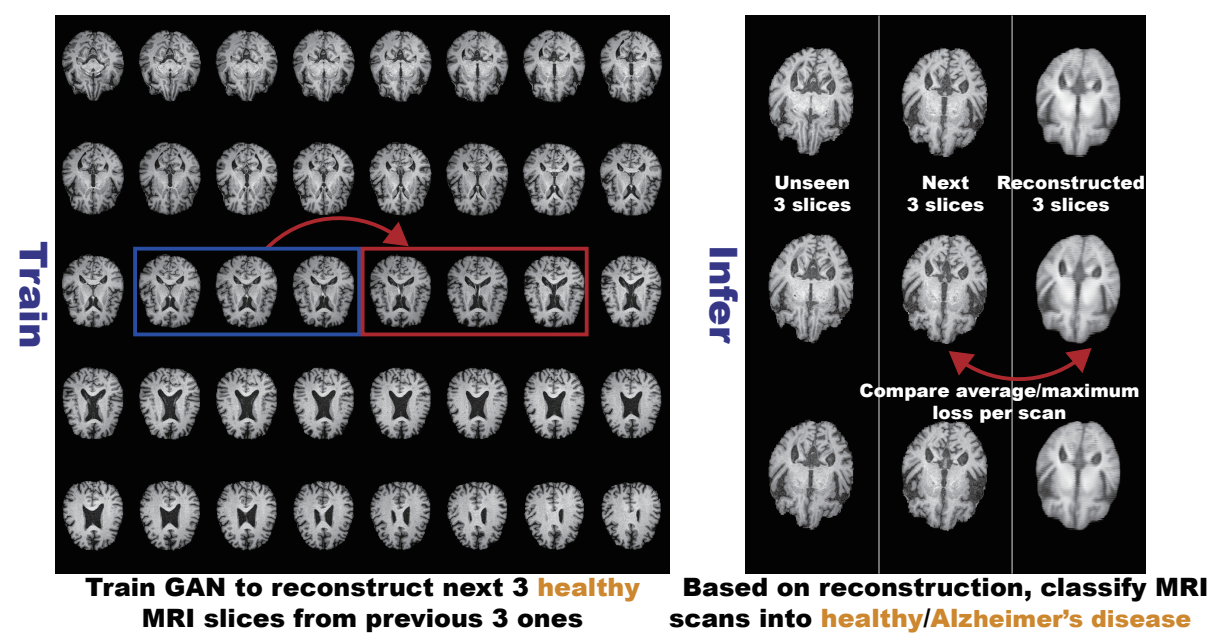

Fig. 1. Unsupervised AD diagnosis framework: we train WGAN-GP $+\ell_{1}$ loss on 3 healthy brain axial MRI slices to reconstruct the next 3 ones, and test it on both unseen healthy and AD cases to classify them based on average/maximum loss (e.g., $\ell_{2}$ loss) per scan.

- Alzheimer's Disease Diagnosis: This first unsupervised AD diagnosis study can reliably detect $\mathrm{AD}$ and also any other diseases.

The remainder of the manuscript is organized as follows: Sect. 2 outlines the state-of-the-art of automated AD diagnosis; Sect. 3 describes the analyzed MRI dataset, as well as the proposed GAN-based unsupervised AD diagnosis framework; experimental results are shown and discussed in Sect. 44 finally, Sect. 5 provides conclusive remarks and future work.

\section{Automated Alzheimer's Disease Diagnosis}

Despite the clinical, social, and economic significance of early AD diagnosisprimarily associated with MCI detection - it generally relies on subjective assessment by physicians (e.g., neurologists, geriatricians, and psychiatrists); to tackle this open challenge, researchers have used classic supervised Machine Learning based on hand-crafted features 1112. More recently, Deep Learning has attracted great attentions owing to its more abstract and descriptive embedding based on multiple non-linear transformations: Liu et al. used a semisupervised CNN to significantly reduce the need for labeled training data[13]; for clinical decision-making, Suk et al. integrated multiple sparse regression models (namely, Deep Ensemble Sparse Regression Network) [14]; Spasov et al. devised a parameter-efficient CNN for 3D separable convolutions, combining dual learning and a specific layer to predict the conversion from MCI to AD within 3 years [15]; 
instead of exploiting the CNNs, Parisot used a semi-supervised Graph Convolutional Network trained on a sub-set of labeled nodes with diagnostic outcomes to represent sparse clinical data [16].

However, to the best of our knowledge, no existing work has conducted fully unsupervised anomaly detection for AD diagnosis since capturing subtle anatomical differences between MCI and AD is challenging.

\section{Materials and Methods}

\subsection{OASIS-3 Dataset}

We use a longitudinal dataset of $176 \times 240 / 176 \times 256$ T1-weighted $(\mathrm{T} 1 \mathrm{w}) 3 \mathrm{~T}$ brain axial MRI slices containing both normal aging subjects/AD patients extracted from the Open Access Series of Imaging Studies-3 (OASIS-3) [17]. The $176 \times 240$ slices are zero-padded to reach $176 \times 256$ pixels. Relying on Clinical Dementia Rating (CDR) [18, common clinical scale for the staging of dementia, the subjects are comprised of:

- Unchanged CDR = 0: Cognitively healthy population;

$-\mathrm{CDR}=0.5$ : Very mild dementia $(\sim \mathrm{MCI})$;

$-\mathrm{CDR}=1:$ Mild dementia;

$-\mathrm{CDR}=2$ : Moderate dementia.

Since our dataset is longitudinal and the same subject's CDRs may vary (e.g., $\mathrm{CDR}=0$ to $\mathrm{CDR}=0.5)$, we only use scans with unchanged CDR $=0$ to assure certainly healthy scans. As CDRs and MRI scans are not always simultaneously acquired, we label MRI scans with CDRs at the closest date. We only select brain MRI slices including hippocampus/amygdala/ventricles among whole 256 axial slices per scan to avoid over-fitting from AD-irrelevant information; the atrophy of the hippocampus/amygdala/cerebral cortex, and enlarged ventricles are strongly associated with $\mathrm{AD}$, and thus they mainly affect the AD classification performance of Machine Learning [19]. Moreover, we discard low-quality MRI slices. The remaining dataset is divided as follows:

- Training set: Unchanged CDR $=0$ (408 subjects/1, 133 scans/57, 834 slices);

- Validation set: Unchanged CDR $=0$ (55 subjects/155 scans/8, 080 slices), $\mathrm{CDR}=0.5$ (53 subjects $/ 85$ scans $/ 4,607$ slices $)$, $\mathrm{CDR}=1(29$ subjects $/ 45$ scans $/ 2,518$ slices $)$, $\mathrm{CDR}=2(2$ subjects $/ 4$ scans $/ 160$ slices $)$;

- Test set: Unchanged CDR $=0$ (113 subjects/318 scans/16, 198 slices), $\mathrm{CDR}=0.5$ (99 subjects $/ 168$ scans $/ 9,206$ slices $)$,

$\mathrm{CDR}=1$ (61 subjects $/ 90$ scans $/ 5,014$ slices $)$,

$\mathrm{CDR}=2(4$ subjects $/ 6$ scans $/ 340$ slices $)$.

The same subject's scans are included in the same dataset. The datasets are strongly biased towards healthy scans similarly to MRI inspection in the clinical routine. During training for reconstruction, we only use the training set containing healthy slices to conduct unsupervised learning. 


\subsection{GAN-based Multiple Adjacent Brain MRI Slice Reconstruction}

To model strong consistency in healthy brain anatomy (Fig. 1), in each scan, we reconstruct the next $3 \mathrm{MRI}$ slices from the previous 3 ones using an image-toimage GAN (e.g., if a scan includes 40 slices $s_{i}$ for $i=1, \ldots, 40$, we reconstruct all possible 35 setups: $\left(s_{i}\right)_{i \in\{1,2,3\}} \mapsto\left(s_{i}\right)_{i \in\{4,5,6\}} ;\left(s_{i}\right)_{i \in\{2,3,4\}} \mapsto\left(s_{i}\right)_{i \in\{5,6,7\}} ; \ldots$; $\left.\left(s_{i}\right)_{i \in\{35,36,37\}} \mapsto\left(s_{i}\right)_{i \in\{38,39,40\}}\right)$. We concatenate adjacent 3 grayscale slices into 3 channels, such as in RGB images. The GAN uses a U-Net-like [20]21] generator with 4 convolutional layers in encoders and 4 deconvolutional layers in decoders respectively with skip connections as well as a discriminator with 3 decoders. We apply batch normalization to both convolution with Leaky Rectified Linear Unit (ReLU) and deconvolution with ReLU. To confirm how reconstructed images' realism and anatomical continuity affect anomaly detection, we compare the GAN models with different loss functions: $(i)$ Dice loss (i.e., a plain U-Net without the discriminator); (ii) WGAN-GP loss; (iii) WGAN-GP loss +100 $\ell_{1}$ loss. Among 8 losses comparing ground truth/reconstructon, average $\ell_{2}$ loss per scan always outperforms the other losses during validation for U-Net and WGAN-GP without/with $\ell_{1}$ loss, and thus we use this loss for testing.

Implementation Details Considering its computational speed, U-Net training lasts for 600,000 steps with a batch size of 64 and both GAN trainings last for 300,000 steps with a batch size of 32 . We use $2.0 \times 10^{-4}$ learning rate for the Adam optimizer 22. The framework is implemented on Keras with TensorFlow as backend.

\subsection{Unsupervised Alzheimer's Disease Diagnosis}

During validation, we compare the following average/maximum losses per scan (i.e., 8 losses) between reconstructed/ground truth 3 slices (Fig. 11) (i) $\ell_{1}$ loss; (ii) $\ell_{2}$ loss; (iii) Dice loss; (iv) Structural Similarity loss. For each model's testing, we separately pick the loss showing the highest AUC between CDR $=0$ (i.e., healthy population) $v s$ all the other CDRs (i.e., dementia) during validation. As a result, we pick the average $\ell_{2}$ loss per scan for all models since squared error is sensitive to outliers and it always outperforms the others. To evaluate its unsupervised AD diagnosis performance for test sets, we show Receiver Operating Characteristics (ROCs)/AUCs between CDR $=0$ vs $(i)$ all the other CDRs; $(i i)$ $\mathrm{CDR}=0.5 ;(i i i) \mathrm{CDR}=1 ;(i v) \mathrm{CDR}=2$. We visualize $\ell_{2}$ loss distributions of $\mathrm{CDR}=0 / 0.5 / 1 / 2$ to know how disease stages affect its discrimination.

\section{Results}

\subsection{Reconstructed Brain MRI Slices}

Fig. 2 illustrates example real MRI slices from test sets and their reconstruction by U-Net and WGAN-GP without/with $\ell_{1}$ loss. The WGAN-GP $+\ell_{1}$ loss can successfully capture T1w-specific appearance and anatomical changes from 

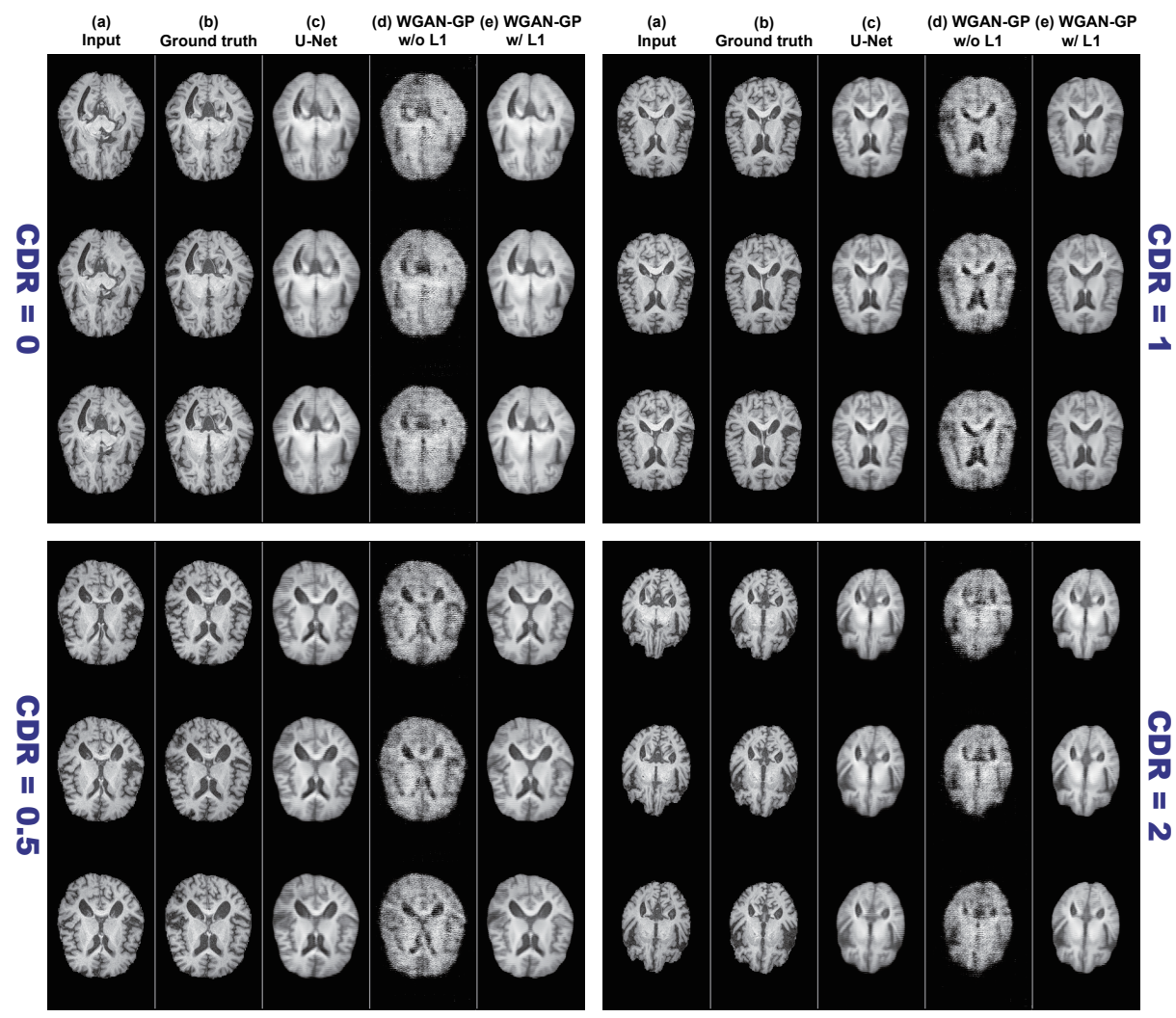

Fig. 2. Example brain MRI slices with $\mathrm{CDR}=0 / 0.5 / 1 / 2$ from test sets: (a) Input 3 real slices; (b) Ground truth next 3 real slices; (c) Next 3 slices reconstructed by U-Net; (d), (e) Next 3 slices reconstructed by WGAN-GP without/with $\ell_{1}$ loss.

the previous 3 slices more smoothly than the U-Net and in more detail than the WGAN-GP without $\ell_{1}$ loss. Since the models are trained only on healthy slices, reconstructing slices with higher CDRs tends to comparatively fail, especially around hippocampus, amygdala, cerebral cortex, and ventricles due to their insufficient atrophy after reconstruction.

\subsection{Unsupervised AD Diagnosis Results}

Fig. 3 shows ROC curves and their AUCs of unsupervised anomaly detection. Since brains with higher CDRs accompany stronger anatomical atrophy from healthy brains, their AUCs between unchanged CDR $=0$ remarkably increase as CDRs increase. Clearly outperforming the other methods in every condition, WGAN-GP $+\ell_{1}$ loss achieves excellent AUCs, especially for higher CDRs - it obtains $\mathrm{AUC}=0.780 / 0.833 / 0.917$ for $\mathrm{CDR}=0$ vs $\mathrm{CDR}=0.5 / 1 / 2$, respectively; this experimental finding derives from $\ell_{1}$ loss' good realism sacrificing 
(a) Unchanged $\mathrm{CDR}=0$ vs $\mathrm{CDR}=0.5+1+2(\mathrm{ROC})$

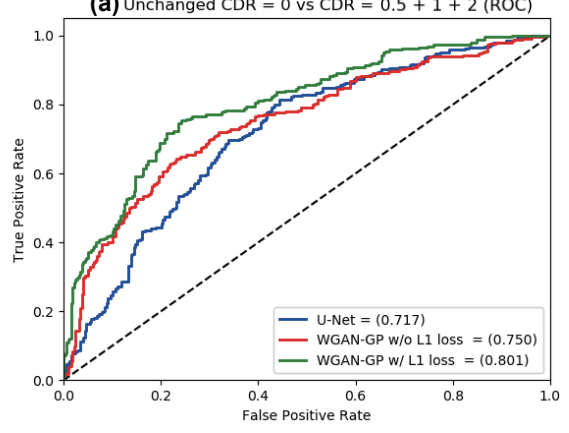

(c) Unchanged $C D R=0$ vs $C D R=1$ (ROC)

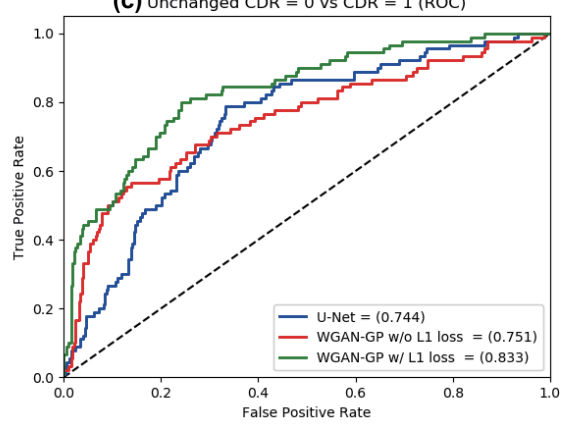

(b) Unchanged $\mathrm{CDR}=0$ vs $\mathrm{CDR}=0.5(\mathrm{ROC})$

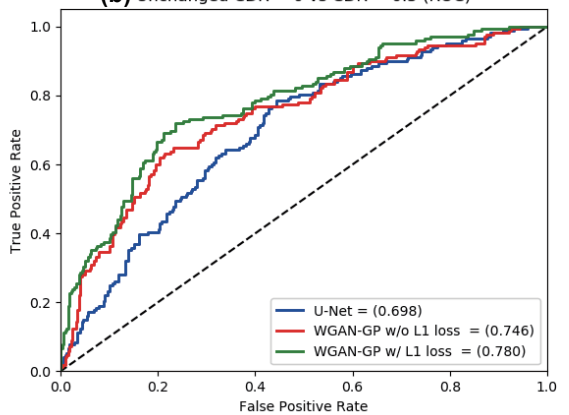

(d) Unchanged $\mathrm{CDR}=0$ vs $\mathrm{CDR}=2$ (ROC)

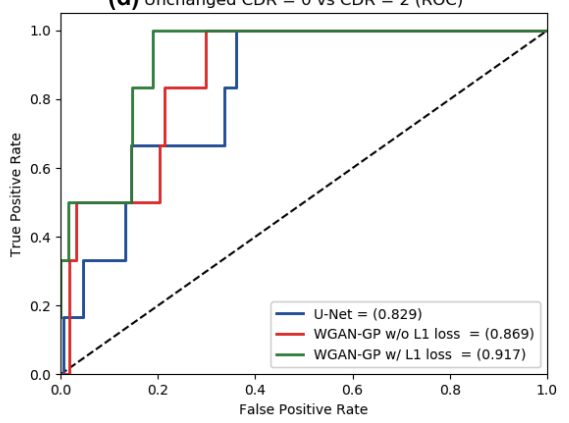

Fig. 3. Unsupervised anomaly detection results using average $\ell_{2}$ loss per scan on reconstructed brain MRI slices (ROCs and AUCs): unchanged CDR $=0$ (i.e., cognitively healthy population) is compared with: (a) all the other CDRs (i.e., dementia); (b) CDR $=0.5$ (i.e., very mild dementia); (c) $\mathrm{CDR}=1$ (i.e., mild dementia); (d) $\mathrm{CDR}=2$ (i.e., moderate dementia).

diversity (i.e., generalizing well only for unseen images with a similar distribution to training images) and WGAN-GP loss' ability to capture recognizable structure. Fig. 4 indicates its good discrimination ability even between healthy subjects vs MCI patients (i.e., $\mathrm{CDR}=0$ vs $\mathrm{CDR}=0.5$ ), which is extremely difficult even in a supervised manner [19]. Interestingly, unlike our visual expectation, WGAN-GP without $\ell_{1}$ loss outperforms plain U-Net regardless of its very blurred reconstruction, showing the superiority of GAN-based reconstruction for diagnosis.

\section{Conclusions and Future Work}

Using a massive amount of healthy images, our GAN-based multiple MRI slice reconstruction can successfully discriminate AD patients from healthy subjects for the first time in an unsupervised manner; our solution leverages a two-step approach: (Reconstruction) $\ell_{1}$ loss generalizes well only for unseen images with 


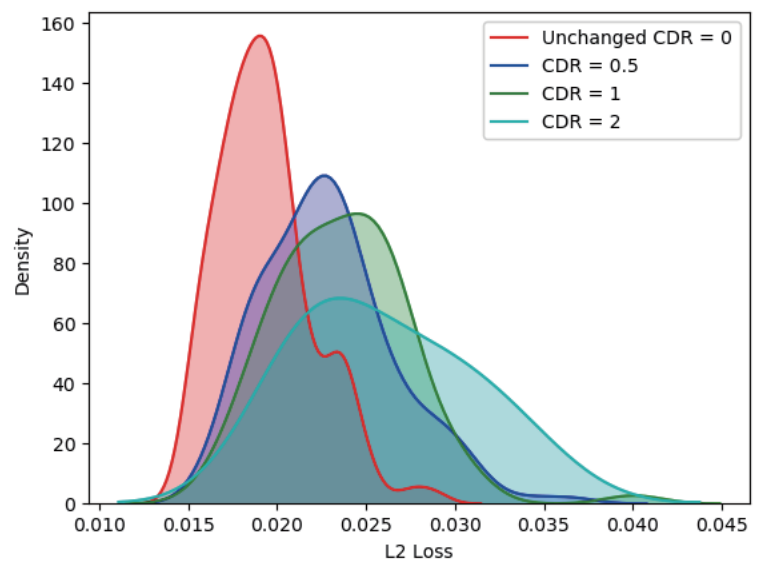

Fig. 4. Distributions of average $\ell_{2}$ loss per scan evaluated on brain MRI slices with $\mathrm{CDR}=0 / 0.5 / 1 / 2$ reconstructed by WGAN-GP $+\ell_{1}$ loss.

a similar distribution to training images while WGAN-GP loss captures recognizable structure; (Diagnosis) $\ell_{2}$ loss clearly discriminates healthy/abnormal data as squared error becomes huge for outliers. Using 1, 133 healthy MRI scans for training, our approach can reliably detect $\mathrm{AD}$ at a very early stage, Mild Cognitive Impairment (MCI), with Area Under the Curve (AUC) 0.780 while detecting $\mathrm{AD}$ at a late stage much more accurately with AUC 0.917-implying its ability to also detect any other diseases.

Accordingly, this first unsupervised anomaly detection across different disease stages reveals that, like physicians' way of diagnosis, large-scale healthy data can reliably aid early diagnosis, such as of MCI, while also detecting late-stage disease much more accurately. Since our method well detects the unseen disease hard-to-detect even in supervised learning, this should also discover/alert any anomalies including rare disease, where supervised learning is inapplicable. As future work, we will reconstruct slices from both previous/next 3 slices (e.g., slices $s_{i}$ for $\left.i=1, \ldots, 9,\left(s_{i}\right)_{i \in\{1,2,3,7,8,9\}} \mapsto\left(s_{i}\right)_{i \in\{4,5,6\}}\right)$ for robustness, also optimizing the number of slices (e.g., 3 slices to 1 or 5 slices). We will investigate more reconstruction networks (e.g., GANs with attention mechanisms) and multiple loss functions for both reconstruction/diagnosis. Lastly, we plan to detect and locate various diseases, including cancer 23] and rare diseases - this work only uses brain MRI slices including hippocampus/amygdala/ventricles for AD diagnosis, but we may have to use all or most brain MRI slices to also detect anomalies appearing in other anatomical locations within the brain. Integrating multimodal imaging data, such as Positron Emission Tomography with specific radiotracers [24, might further improve AD diagnosis [25], even when analyzed modalities are partially unavailable [26]. 


\section{Acknowledgment}

This research was supported by AMED Grant Number JP18lk1010028, and also partially supported by The Mark Foundation for Cancer Research and Cancer Research UK Cambridge Centre [C9685/A25177]. Additional support has been provided by the National Institute of Health Research (NIHR) Cambridge Biomedical Research Centre. Zoltán Ádám Milacski was supported by Grant Number VEKOP-2.2.1-16-2017-00006. The OASIS-3 dataset has Grant Numbers P50 AG05681, P01 AG03991, R01 AG021910, P50 MH071616, U24 RR021382, and R01 MH56584.

\section{References}

1. Goodfellow, I., Pouget-Abadie, J., Mirza, M., Xu, B., Warde-Farley, D., Ozair, S., et al.: Generative adversarial nets. In: Proceedings of Advances in Neural Information Processing Systems (NIPS). (2014) 2672-2680

2. Frid-Adar, M., Diamant, I., Klang, E., Amitai, M., Goldberger, J., Greenspan, H.: GAN-based synthetic medical image augmentation for increased CNN performance in liver lesion classification. Neurocomputing 321 (2018) 321-331

3. Han, C., Rundo, L., Araki, R., Nagano, Y., Furukawa, Y., et al.: Combining noiseto-image and image-to-image GANs: brain MR image augmentation for tumor detection. IEEE Access 7(1) (2019) 156966-156977

4. Han, C., Kitamura, Y., Kudo, A., Ichinose, A., Rundo, L., Furukawa, Y., et al.: Synthesizing diverse lung nodules wherever massively: 3D multi-conditional GANbased CT image augmentation for object detection. In: Proc. International Conference on 3D Vision (3DV). (2019) 729-737

5. Han, C., Murao, K., Noguchi, T., et al.: Learning more with less: Conditional PGGAN-based data augmentation for brain metastases detection using highlyrough annotation on MR images. In: Proc. ACM International Conference on Information and Knowledge Management (CIKM). (2019) 119-127

6. Schlegl, T., Seeböck, P., Waldstein, S.M., Langs, G., Schmidt-Erfurth, U.: fAnoGAN: fast unsupervised anomaly detection with generative adversarial networks. Med. Image Anal. 54 (2019) 30-44

7. Uzunova, H., Schultz, S., Handels, H., Ehrhardt, J.: Unsupervised pathology detection in medical images using conditional variational autoencoders. Int. J. Comput. Assist. Radiol. Surg. 14(3) (2019) 451-461

8. Chen, X., Konukoglu, E.: Unsupervised detection of lesions in brain MRI using constrained adversarial auto-encoders. In: International Conference on Medical Imaging with Deep Learning (MIDL). (2018) arXiv preprint arXiv:1806.04972.

9. Gulrajani, I., Ahmed, F., Arjovsky, M., Dumoulin, V., Courville, A.C.: Improved training of Wasserstein GANs. In: Advances in Neural Information Processing Systems. (2017) 5769-5779

10. Han, C., Hayashi, H., Rundo, L., Araki, R., Shimoda, W., Muramatsu, S., et al.: GAN-based synthetic brain MR image generation. In: Proc. International Symposium on Biomedical Imaging (ISBI), IEEE (2018) 734-738

11. Salvatore, C., Cerasa, A., Battista, P., Gilardi, M.C., Quattrone, A., Castiglioni, I.: Magnetic resonance imaging biomarkers for the early diagnosis of Alzheimer's disease: a machine learning approach. Front. Neurosci. 9 (2015) 307 
12. Nanni, L., Brahnam, S., Salvatore, C., Castiglioni, I.: Texture descriptors and voxels for the early diagnosis of Alzheimer's disease. Artif. Intell. Med. 97 (2019) $19-26$

13. Liu, S., Liu, S., Cai, W., Pujol, S., Kikinis, R., Feng, D.: Early diagnosis of alzheimer's disease with deep learning. In: Proc. International Symposium on Biomedical Imaging (ISBI), IEEE (2014) 1015-1018

14. Suk, H.I., Lee, S.W., Shen, D.: Deep ensemble learning of sparse regression models for brain disease diagnosis. Med. Image Anal. 37 (2017) 101-113

15. Spasov, S., Passamonti, L., Duggento, A., Liò, P., Toschi, N., Initiative, A.D.N., et al.: A parameter-efficient deep learning approach to predict conversion from mild cognitive impairment to Alzheimer's disease. NeuroImage 189 (2019) 276-287

16. Parisot, S., Ktena, S.I., Ferrante, E., Lee, M., Guerrero, R., Glocker, B., Rueckert, D.: Disease prediction using graph convolutional networks: application to autism spectrum disorder and Alzheimer's disease. Med. Image Anal. 48 (2018) 117-130

17. LaMontagne, P.J., Keefe, S., Lauren, W., et al.: OASIS-3: longitudinal neuroimaging, clinical, and cognitive dataset for normal aging and Alzheimer's disease. Alzheimers Dement. 14(7) (2018) P1097

18. Morris, J.C.: The Clinical Dementia Rating (CDR): current version and scoring rules. Neurology 43(11) (1993) 2412-2414

19. Ledig, C., Schuh, A., Guerrero, R., Heckemann, R.A., Rueckert, D.: Structural brain imaging in Alzheimer's disease and mild cognitive impairment: biomarker analysis and shared morphometry database. Sci. Rep. 8 (2018) 11258

20. Ronneberger, O., Fischer, P., Brox, T.: U-Net: Convolutional networks for biomedical image segmentation. In: Medical Image Computing and Computer-Assisted Intervention (MICCAI). Volume 9351 of LNCS., Springer (2015) 234-241

21. Rundo, L., Han, C., Nagano, Y., et al.: USE-Net: incorporating squeeze-andexcitation blocks into U-Net for prostate zonal segmentation of multi-institutional MRI datasets. Neurocomputing 365 (2019) 31-43

22. Kingma, D.P., Ba, J.: Adam: A method for stochastic optimization. arXiv preprint arXiv:1412.6980 (2014)

23. Rundo, L., Militello, C., Russo, G., Vitabile, S., Gilardi, M.C., Mauri, G.: GTVcut for neuro-radiosurgery treatment planning: an MRI brain cancer seeded image segmentation method based on a cellular automata model. Nat. Comput. 17 (2018) $521-536$

24. Rundo, L., Stefano, A., Militello, C., Russo, G., Sabini, M.G., D’Arrigo, C., Marletta, F., Ippolito, M., Mauri, G., Vitabile, S., Gilardi, M.C.: A fully automatic approach for multimodal PET and MR image segmentation in Gamma Knife treatment planning. Comput. Methods Programs Biomed. 144 (2017) 77-96

25. Brier, M.R., Gordon, B., Friedrichsen, K., McCarthy, J., Stern, A., Christensen, J., Owen, C., Aldea, P., Su, Y., Hassenstab, J., et al.: Tau and A $\beta$ imaging, CSF measures, and cognition in Alzheimer's disease. Sci. Trans. Med. 8(338) (2016) 338ra66-338ra66

26. Li, R., Zhang, W., Suk, H.I., Wang, L., Li, J., Shen, D., Ji, S.: Deep learning based imaging data completion for improved brain disease diagnosis. In: Medical Image Computing and Computer-Assisted Intervention (MICCAI). Volume 8675 of LNCS., Springer (2014) 305-312 